\title{
Postural tachycardia and orthostatic intolerance: chicken, egg or unrelated?
}

\author{
Mojca Kirbiš • Fajko F. Bajrović • Bernard Meglič
}

Received: 1 October 2013/ Accepted: 23 October 2013/Published online: 6 November 2013

(C) Springer-Verlag Berlin Heidelberg 2013

\section{Dear Editor,}

We have read with interest the paper Orthostatic intolerance without postural tachycardia-how much dysautonomia? recently published in CAR. It is perhaps of relevance to readers that we arrived at similar findings regarding the presentation of orthostatic intolerance in our study, although with a different design and in a geographically distant environment [1]. Similarly to Parsaik et al., we compared patients with persistent orthostatic symptoms with and without postural tachycardia and found no difference in demographic or clinical characteristics between groups (for reasons explained in the cited paper, a relatively lower cut-off for postural tachycardia was used). Furthermore, in our as yet unpublished work, no differences were found in heart rate variability, peripheral vasoconstriction, or serum hormone levels. We can therefore only agree with the Mayo Clinic group that orthostatic intolerance without postural tachycardia is a condition encountered in everyday practice and a relevant research topic.

Regards,

Mojca Kirbiš

\section{References}

1 Kirbiš M, Bajrović FF, Čižek-Sajko M, Meglič B, Grad A (2013) Postural tachycardia syndrome during head up tilt test predicts orthostatic intolerance in young adults. Zdrav Vestn 82:386-394

M. Kirbiš $(\bowtie)$ · F. F. Bajrović · B. Meglič

University Medical Centre Ljubljana, Ljubljana, Slovenia

e-mail: mojca.kirbis@kclj.si

\section{Kirbiš}

General Hospital Nova Gorica, Nova Gorica, Slovenia

F. F. Bajrović

Medical Faculty, University of Ljubljana, Ljubljana, Slovenia 